\title{
Differential susceptibility to the environment: Toward an understanding of sensitivity to developmental experiences and context
}

\author{
BRUCE J. ELLIS $^{a}$ AND W. THOMAS BOYCE ${ }^{b}$ \\ ${ }^{a}$ University of Arizona; and ${ }^{b}$ University of British Columbia
}

Science, like evolution, is often remarkably convergent in its generativity of new ideas and its exploration of novel conceptual territory. Just as evolution has repeatedly converged upon common phenotypic solutions to problems of survival and reproduction among species of differing lineage (Morris, 2010), the history of scientific inquiry, and that of developmental science in particular, has also been marked by concurrent and homologous discovery by a sometimes striking simultaneity in its arrival at shared theoretical insights along paths of differing origins and trajectories. Thus, it has been with the emergence of "differential susceptibility to the environment": a construct—really, a shared solution to a set of compelling conceptual and empirical dilemmas-that forms the centerpiece of this Special Section of Development and Psychopathology.

Inspired by a provocative and broadly attended symposium ("Do Children Vary in Their Plasticity? Differential Susceptibility to Rearing Experiences") at the 2009 Annual Meeting of the Society for Research in Child Development (SRCD), the Special Section explores the convergence of two theories conceptualizing reactivity to environmental stimuli as an indicator of sensitivity or susceptibility to environmental influence. The "biological sensitivity to context" theory advanced by Boyce and Ellis (2005; see also Boyce

Address correspondence and reprint requests to: Bruce J. Ellis, Norton School of Family and Consumer Sciences, University of Arizona, McClelland Park, 650 North Park Avenue, Tucson, AZ 85721-0078; E-mail: bjellis @email.arizona.edu; or W. Thomas Boyce, College for Interdisciplinary Studies and Faculty of Medicine, 440-2206 East Mall, University of British Columbia, Vancouver, BC V6T 1Z3, Canada; E-mail: tom.boyce@ubc.ca. et al., 1995) originated in empirical observations of differences in children's autonomic and adrenocortical reactivity to challenge and posited a context-sensitive endophenotype rendering a subset of children unusually susceptible to the risk-inducing and development-enhancing influences of both negative and positive early social environments. At the same time, the "differential susceptibility" theory posed by Belsky $(1997,2005)$ started with the question "Why should childhood experiences influence later development?" Based on reflections prompted by this question, it hypothesized that children should differ in their susceptibility to rearing environments as a bet-hedging strategy against an uncertain future. Both theories converged on evolutionary explanations of why and how individuals vary systematically in their sensitivity or "permeability" to experiential and contextual influences on development and health.

Most students of child development do not presume that all children are equally susceptible to rearing and other contextual experiences; a long history of research on Parenting $\times$ Temperament interactions clearly suggests otherwise. Nevertheless, much work still focuses on contextual effects that apply equally to all children and thus fails to consider the possibility that whether, how, and to what degree early experiences influence child development may critically depend upon individual characteristics. All four empirical papers presented at the SRCD symposium examined the extent to which such child characteristics moderate effects of early rearing experiences on children's adjustment and development. Drawing on biological sensitivity to context or differential susceptibility theory, each paper focused on a phenotypic, endophenotypic, or genotypic marker of reactivity as a moderator of susceptibility to rearing influence. In the first two papers the moderator was biological 
reactivity to stress, in the third it was negative emotional reactivity, and in the fourth it was variation in the dopamine receptor D4 (DRD4) gene.

The Special Section merges the four SRCD papers with six others (two theoretical and four empirical) that together consider the possibility that children scoring higher on a measured reactivity marker will prove more susceptible to both positive and negative early environments, for better and for worse. The theoretical papers establish the conceptual basis for such a claim, and the introductory theoretical paper brings together the primary architects of the theories of biological sensitivity to context and differential susceptibility (Ellis, Boyce, Belsky, Bakermans-Kranenburg, \& van IJzendoorn, 2011) in an attempt to construct an integrated vision of the field. Each of the empirical papers then presents crossover interactions showing disproportionately high rates of mental and physical health problems among reactive children and adolescents raised in adverse environments and unusually low rates (or unusually high rates of prosocial behavior, such as donating to charity) among such individuals raised in low-stress, supportive settings. We argue that these findings have considerable implications for understanding how individuals respond to all kinds of environmental conditions and may be critically important for understanding the developmental consequences of salient, early rearing conditions, as well as guiding the creation of early preventive interventions.

In total, the emerging theory of differential susceptibility not only reinterprets much of what is known about rearing influences on behavioral adjustment and health, affording a new perspective on Person $\times$ Environment interactions in development, but also generates novel hypotheses and suggests new lines of research to be followed (Ellis, Boyce, et al., 2011). Accordingly, the theory raises many issues and questions for developmental scientists. Here we outline a set of the critical issues and questions that are addressed in the Special Section.

\section{Neurobiological Susceptibility: From Genotype to Endophenotype to Behavior}

A major goal of research on differential susceptibility to the environment is uncovering a high-resolution map of the mechanisms of context sensitivity. Differences in openness to environmental influence are underpinned by neurobiological variation in sensitivity to contextual signals and cues and instantiated through multiple genetic polymorphisms, endophenotypes, and behaviors, as illustrated by the series of empirical articles in the Special Section. Bakermans-Kranenburg and van IJzendoorn (2007) suggest that endophenotypic variation in environmental susceptibility might be underpinned by allelic variation in the genes that guide development of the dopaminergic circuitry of the brain, which in turn governs thresholds of responsiveness to reward and punishment. The research presented in this Special Section demonstrates that polymorphisms involving dopamine-related genes (DRD2, DRD4, dopamine transporter 1) are associated with either highly adaptive or suboptimal child and adult developmental endpoints, depending upon rearing experiences and life events, and offers empirical support for such a mechanism (Bakermans-Kranenburg \& van IJzendoorn, 2011; Knafo, Israel, \& Ebstein, 2011). In an additional, novel test of a parallel hypothesis, polymorphic variation in the gene encoding estrogen receptor- $\alpha$ is examined for the first time in a paper by Manuck, Craig, Flory, Halder, and Ferrell (2011) as a possible, promising marker of neurobiological sensitivity to context.

Further genetic and epigenetic processes in humans, which involve the developmental shaping of neurotransmitter and molecular signaling pathways, appear to regulate brain structures mediating reward, fear, and emotional reactivity (Feder, Nestler, \& Charney, 2009). Thus, at the level of neural function, differential susceptibility to environmental exposures may be determined by systematic differences in the functioning of specific brain circuitry; neuronal activity; and neurotransmitter production, processing, and metabolism. In a pioneering article in the Special Section, Whittle et al. (2011) present data suggesting not only that differences in hippocampal volume may be related to vulnerability in adverse environmental contexts but also that the same volumetric characteristics may predict positive outcomes in the context of favorable environments. The authors suggest that the hippocampus could serve as a biological substrate for sensitivity to context through its roles in processing and integrating information from the environment with learning and memory processes.

According to the biological sensitivity to context theory (Boyce \& Ellis, 2005), neurobiological susceptibility is also embodied in the differential reactivity of the two peripheral, neuroendocrine stress response systems. There is extensive individual variability in the responsivity of these systems to standardized laboratory challenges, and individuals with heightened reactivity in either or both of the autonomic or adrenocortical systems appear to sustain the worst or the best of the observed health and developmental outcomes, contingent upon the level of adversity or support prevalent in rearing environments. The role of stress reactivity in moderating the effects of family and school environments on child and adolescent health outcomes is examined in several papers in the Special Section (Ellis, Shirtcliff, et al., 2011; Essex, Armstrong, Burk, Goldsmith, \& Boyce, 2011; Obradović, Bush, \& Boyce, 2011).

Finally, neurobiological susceptibility to the environment must be manifest through behavioral interactions with the environment. Most behavioral, phenotypic markers of susceptibility that have been identified involve negative emotionality or reactivity in one form or another (Belsky, 2005). Based on work with rhesus monkeys, Suomi (1997) suggests that the timidity of behaviorally inhibited, neophobic infants affords them extensive opportunity to learn by watching. Likewise, Kochanska, Askan, and Joy (2007) propose that the ease with which anxiety is induced in reactive, fearful children helps them to respond favorably to gentle parental discipline but be negatively affected by harsh discipline. Two articles in the Special Section examine the role of child behavioral reactivity in moderating the effects of social context on developmental outcomes. Essex et al. (2011) provide evidence that 
temperamental inhibition/disinhibition increases susceptibility to teacher-child relationships; Cassidy, Woodhouse, Sherman, Stupica, and Lejuez (2011) find that infant irritability enhances sensitivity to maternal behavior.

\section{Toward an Understanding of Different Domains of Neurobiological Susceptibility}

The question of domain specificity in neurobiological susceptibility to the environment is a core dilemma within theory and research on context sensitivity. Are some individuals more susceptible than others to a wide and diverse array of developmental inputs, thereby influencing a wide and diverse array of developmental outcomes? Or are some individuals, for example, highly susceptible to parental influences on academic achievement whereas others prove highly susceptible to peer effects on risk-taking behavior?

The papers by Obradović et al. (2011) and Essex et al. (2011) help to define this important and heuristic "growing edge" of the differential susceptibility construct, together challenging context sensitivity as a monolithic category and proposing a more differentiated and nuanced vision of its operation within human development. Obradović and colleagues note some findings that are precisely the opposite of those suggested by biological sensitivity to context or differential susceptibility theory, and they examine the interactions between the specific challenge of marital conflict exposure and autonomic reactivity to laboratory challenges in predicting children's internalizing and externalizing behavior problems. Although hypothesized interactions are confirmed, the direction of such effects vary with the character of the task that was utilized to evoke physiological responses, suggesting a need to consider the question "reactivity to what?" and to move beyond single, unidimensional measures of "reactivity." The paper by Essex and colleagues similarly expands our vision of reactivity to a consideration of both biological and behavioral measures and further demonstrates that sensitivity to social context depends on the temperamental characteristics of the child. Their results argue that an individual child's sensitivity is attuned to particular dimensions of the social environment in a manner that links the salience of contextual dimensions to the temperamental "receptivity" of the child. Taken together, the two papers demand a more subtle and refined conceptualization of reactivity, biological sensitivity, and differential susceptibility.

\section{Experimental Tests of Differential Susceptibility}

Although there is considerable correlational data that provide support for differential susceptibility to social contexts, compelling experimental evidence of environmental effects being moderated by temperamental, physiological, and/or genetic factors remains limited. Experimental examination of neurobiological susceptibility by means of intervention affords a more solid basis for causal inference. Specifically, experimental designs where the environment is an intervention or control condition to which participants are randomly assigned overcome some of the limitations of correlational studies of Gene $\times$ Environment or Temperament $\times$ Parenting interactions and allow for stronger conclusions about the direction of effects (see Bakermans-Kranenburg, van IJzendoorn, Pijlman, Mesman, \& Juffer, 2008; Cicchetti \& Gunnar, 2008).

Past experimental research to enhance parenting has shown that highly negatively reactive infants profited most in terms of attachment security (Klein Velderman, Bakermans-Kranenburg, Juffer, \& Van IJzendoorn, 2006). Building on this prior work, Cassidy and colleagues (2011) use a randomized controlled trial design to examine how infant irritability and maternal attachment style work together as moderators of the efficacy of an attachment-based intervention. In doing so, they seek and find evidence of differential susceptibility to rearing influences. This type of experimental work advances knowledge by disconfirming a critical alternative explanation: that differential susceptibility is driven by the effects of child characteristics on rearing experiences.

\section{The Bright Side of Differential Susceptibility}

Because the essence of the differential susceptibility model is that individuals who display high neurobiological susceptibility to the environment are not only disproportionately affected by negative contexts (the dark side) but also respond more favorably to positive environmental influences (the bright side), it is imperative that tests of this hypothesis secure adequate variance in environmental conditions and behavioral outcomes (Ellis, Essex, \& Boyce, 2005). By targeting risky environments and behaviors, many studies of developmental psychopathology may obscure the potential benefits of exposure to positive contexts for susceptible individuals. For example, genetic studies of neurobiological susceptibility have typically focused on the interaction between specific "risk" or "susceptibility" alleles and adverse rearing environments, but positive outcomes of the same genes interacting with positive rearing environments are still understudied.

Several articles in the Special Section redress this issue by presenting new empirical evidence for the bright side of Gene $\times$ Environment and Temperament $\times$ Parenting interactions. Bakermans-Kranenburg and van IJzendoorn (2011) find that children with secure attachment representations, as indicated by telling stories that describe positive feelings and harmonious interactions with their mothers, donated more money to charity, but only if they had the DRD4 7-repeat allele. In a broader sense, the authors examine the Gene $\times$ Environment literature on children up to 10 years of age to assess whether differential genetic susceptibility can be generalized to other domains of development and to a wider set of dopamine-system related genes than DRD4. Their meta-analysis supports "for better and for worse" effects, providing the most systematic evidence to date that more susceptible children profit the most from positive environments.

The paper by Knafo and colleagues (2011) also provides strong support of the principle that differential susceptibility 
works bidirectionally, predicting both the most positive and the least positive outcomes of development. Genetically susceptible children, again indexed by the DRD4 7-repeat allele, showed the highest level of prosocial behavior when receiving highly positive parenting but the lowest levels when their mothers were low in warmth and positivity. It is intriguing that this extreme sensitivity may cause children to behave prosocially for the wrong reasons. Genetically susceptible children whose mothers tended to use punishment without explanation reacted prosocially more than other children did, possibly because they try to avoid punishment as much as possible, because they are more sensitive to rewards. Finally, Cassidy et al. (2011) demonstrate that a parenting intervention to improve maternal sensitivity enhances attachment security in infants, but only among susceptible children, as indicated by observed infant irritability.

\section{Developmental Influences on Neurobiological Susceptibility}

The ontogenetic origins of differential susceptibility and, more specifically, identifying specific genetic and environmental influences on heightened susceptibility to environmental influence, is another key issue engaged in the Special Section (see especially Ellis, Boyce, et al., 2011). Boyce and Ellis' (2005) theory of biological sensitivity to context advanced the claim that physiological reactivity, a key marker of neurobiological susceptibility, arises in response to both very positive and very negative childhood environments (i.e., the Ushaped curve). By contrast, Belsky's (1997, 2005) original differential susceptibility hypothesis was based on the presumption that differences in context sensitivity were more or less genotypic in origin. However, neither perspective precluded the alternative.

One implication of the biological sensitivity to context model is that biobehavioral reactivity to stress should be at least partially influenced by prenatal experiences. The literature on "prenatal programming" has focused principally on how maternal stress during pregnancy adversely influences the fetus and developing child, thereby laying the groundwork for a host of untoward health consequences, including metabolic diseases in middle age. In their article for the Special Section, Pluess and Belsky (2011) offer a contrasting view; they hypothesize that maternal stress fosters negative emotionality in infancy and physiological reactivity and thus heightens susceptibility to postnatal environmental influences, whether they are positive or negative in character. Old and new evidence consistent with this proposition is reviewed, leading to the conclusion that there may be "prenatal programming of postnatal plasticity."

\section{Addressing the Nature-Nurture Debate: The Case of Pubertal Timing}

An important impetus for the development of Belsky's (1997) differential susceptibility theory was the behavior-genetic critique of much socialization research, a critique that was eventually directed at Belsky, Steinberg, and Draper's (1991) evolutionary theory of socialization (Rowe, 2000; Rowe, Vazsonyi, \& Figueredo, 1997). Belsky et al. (1991) attempted to recast thinking about early experience in terms of the developmental regulation of reproductive strategy. Central to the theory was the conditional adaptation-based claim that stressful and supportive extrafamilial environments influence family dynamics, especially parent-child and parent-parent relationships, thereby shaping children's early emotional and behavioral development and, through it, subsequent reproductive development and fitness. Behavior-genetic analyses suggested instead that variation in children's emotional and behavioral development and subsequent reproductive strategies was not so much attributable to family experiences but to genes shared by parents and children, and thus genetic mediation.

Differential susceptibility offered a potential resolution to this debate. If children differ in whether and how much they are affected by rearing experiences, then both the behavior genetic and socialization perspectives could be correct, but for different individuals (Belsky, 2000). That is, the main effects of familial and ecological conditions on sexual development may overestimate the impact of those conditions in some children (low susceptibility, more fixed reproductive development, genetic mediation) and underestimate it in others (high susceptibility, more plastic reproductive development, environmental mediation).

Two studies in the Special Section, which use very different methods and measures of neurobiological susceptibility to the environment, test this hypothesis (Ellis, Shirtcliff, et al., 2011; Manuck et al., 2011). Consistent with past research, both studies document small main effects of family processes on pubertal timing or tempo. Most critically, however, these main effects are moderated by the measured indices of neurobiological susceptibility, including variation in physiological stress responsivity (Ellis, Shirtcliff, et al., 2011) and polymorphic variation in estrogen receptor- $\alpha$ (Manuck et al., 2011). In both studies, more susceptible individuals are especially open to the development-enhancing and risk-promoting effects of family environments on pubertal development. These results highlight the importance of both nature (for some children) and nurture (for others).

\section{Conclusion}

The papers comprising this Special Section collectively offer a comprehensive and challenging topography of a new, largely uncharted territory in the landscape of research on developmental psychopathology. That territory reveals the demonstrable variation (genotypic, endophenotypic, and behavioral) in children's susceptibility to environmental influences and rearing conditions. The series of 10 theoretical and empirical papers traces the perimeter of the differential susceptibility construct; explores the operationalization of the construct at varied levels of biological abstraction; harvests a rich array of evidence for the double-edged, risk-amplifying, and protectionenhancing implications of such sensitivity; and sketches a 
broad expanse of issues, questions, and conundrums for future study. Thus, the papers also yield a pleasing and inspiring assembly of convergences and reconciliations. In a mimicking of evolutionary convergence, two homologous theories of distinctive origins (biological sensitivity to context and differential susceptibility) arrive at a common and mutually supportive conceptual destination. By invoking the evolutionary constructs of

\section{References}

Bakermans-Kranenburg, M. J., \& van IJzendoorn, M. H. (2007). Genetic vulnerability or differential susceptibility in child development: The case of attachment [Research review]. Journal of Child Psychology and Psychiatry, 48, 1160-1173.

Bakermans-Kranenburg, M. J., \& van IJzendoorn, M. H. (2011). Differential susceptibility to rearing environment depending on dopamine-related genes: New evidence and a meta-analysis. Development and Psychopathology, 23, 39-52.

Bakermans-Kranenburg, M. J., van IJzendoorn, M. H., Pijlman, F. T. A., Mesman, J., \& Juffer, F. (2008). Differential susceptibility to intervention: Dopamine D4 receptor polymorphism (DRD4 VNTR) moderates effects on toddlers' externalizing behavior in a randomized control trial. Developmental Psychology, 44, 293-300.

Belsky, J. (1997). Variation in susceptibility to rearing influences: An evolutionary argument. Psychological Inquiry, 8, 182-186.

Belsky, J. (2000). Conditional and alternative reproductive strategies: Individual differences in susceptibility to rearing experience. In J. Rodgers, D. Rowe, \& W. Miller (Eds.), Genetic influences on human fertility and sexuality: Theoretical and empirical contributions from the biological and behavioral sciences (pp. 127-146). Boston: Kluwer.

Belsky, J. (2005). Differential susceptibility to rearing influences: An evolutionary hypothesis and some evidence. In B. Ellis \& D. Bjorklund (Eds.), Origins of the social mind: Evolutionary psychology and child development (pp. 139-163). New York: Guilford Press.

Belsky, J., Steinberg, L., \& Draper, P. (1991). Childhood experience, interpersonal development and reproductive strategy: An evolutionary theory of socialization. Child Development, 62, 647-670.

Boyce, W. T., Chesney, M., Alkon, A., Tschann, J. M., Adams, S., Chesterman, B., et al. (1995). Psychobiologic reactivity to stress and childhood respiratory illnesses: Results of two prospective studies. Psychosomatic Medicine, 57, 411-422.

Boyce, W. T., \& Ellis, B. J. (2005). Biological sensitivity to context: I. An evolutionary-developmental theory of the origins and functions of stress reactivity. Development and Psychopathology, 17, 271-301.

Cassidy, J., Woodhouse, S. S., Sherman, L. J., Stupica, B., \& Lejuez, C. W. (2011). Enhancing infant attachment security: An examination of treatment efficacy and differential susceptibility. Development and Psychopathology, 23, 131-148.

Cicchetti, D., \& Gunnar, M. R. (2008). Integrating biological measures into the design and evaluation of preventive interventions. Development and Psychopathology, 20, 737-743.

Ellis, B. J., Boyce, W. T., Belsky, J., Bakermans-Kranenburg, M. J., \& van IJzendoorn, M. H. (2011). Differential susceptibility to the environment: An evolutionary-neurodevelopmental theory. Development and Psychopathology, 23, 7-28.

Ellis, B. J., Essex, M. J., \& Boyce, W. T. (2005). Biological sensitivity to context: II. Empirical explorations of an evolutionary-developmental theory. Development and Psychopathology, 17, 303-328. bet-hedging and conditional adaptation, this new, synthetic theory of differential susceptibility comprises a set of shared, unifying principles that may guide future work on environmental effects in both developmental science and evolutionary biology. By revealing such principles, we hope the collected papers effect a congenial and possibly useful reconciliation between the risk- and protection-centered divisions of our field.

Ellis, B. J., Shirtcliff, E. A., Boyce, W. T., Deardorff, J., \& Essex, M. J. (2011). Quality of early family relationships and the timing and tempo of puberty: Effects depend on biological sensitivity to context. Development and Psychopathology, 23, 85-99.

Essex, M. J., Armstrong, J. M., Burk, L. R., Goldsmith, H. H., \& Boyce, W. T. (2011). Biological sensitivity to context moderates the effects of the early teacher-child relationship on the development of mental health by adolescence. Development and Psychopathology, 23, 149-161.

Feder, A., Nestler, E. J., \& Charney, D. S. (2009). Psychobiology and molecular genetics of resilience. Nature Reviews Neuroscience, 10, 446-457.

Klein Velderman, M., Bakermans-Kranenburg, M. J., Juffer, F., \& van IJzendoorn, M. H., (2006). Effects of attachment-based interventions on maternal sensitivity and infant attachment: Differential susceptibility of highly reactive infants. Journal of Family Psychology, 20, 266-274.

Knafo, A., Israel, S., \& Ebstein, R. P. (2011). Heritability of children's prosocial behavior and differential susceptibility to parenting by variation in the dopamine receptor D4 gene. Development and Psychopathology, 23, 53-67.

Kochanska, G., Askan, N., \& Joy, M. E. (2007). Children's fearfulness as a moderator of parenting in early socialization: Two longitudinal studies. Developmental Psychology, 43, 222-237.

Manuck, S. B., Craig, A. E., Flory, J. D., Halder, I., \& Ferrell, R. E. (2011). Reported early family environment covaries with menarcheal age as a function of polymorphic variation in estrogen receptor- $\alpha$. Development and Psychopathology, 23, 69-83.

Morris, S. C. (2010). Evolution: Like any other science it is predictable. Philosophical Transactions of the Royal Society of London B, 365, 133-145.

Obradović, J., Bush, N. R., \& Boyce, W. T. (2011). The interactive effect of marital conflict and stress reactivity on externalizing and internalizing symptoms: The role of laboratory stressors. Development and Psychopathology, 23, 101-114.

Pluess, M., \& Belsky, J. (2011). Prenatal programming of postnatal plasticity? Development and Psychopathology, 23, 29-38.

Rowe, D. C. (2000). Environmental and genetic influences on pubertal development: Evolutionary life history traits? In J. L. Rodgers, D. C Rowe, \& W. B. Miller (Eds.), Genetic influences on human fertility and sexuality: Recent empirical and theoretical findings (pp. 147-168). Boston: Kluwer.

Rowe, D. C., Vazsonyi, A. T., \& Figueredo, A. J. (1997). Mating-effort in adolescence: A conditional or alternative strategy. Personality and Individual Differences, 23, 105-115.

Suomi, S. (1997). Early determinants of behaviour. British Medical Bulletin, 53, 170-184.

Whittle, S., Yap, M. B. H., Sheeber, L., Dudgeon, P., Yücel, M., Pantelis, C., et al. (2011). Hippocampal volume and sensitivity to maternal aggressive behavior: A prospective study of adolescent depressive symptoms. Development and Psychopathology, 23, 115-129. 